\title{
Aprendizaje basado en problemas para el pensamiento crítico y creativo en estudiantes de educación primaria
}

\author{
Mg. Erica Paola Chimoy Lenz \\ chimoyle@ucvvirtual.edu.pe \\ https://orcid.org/0000-0002-2935-4502 \\ Programa Académico de Doctorado en Educación \\ Escuela de postgrado \\ Universidad César Vallejo \\ Chiclayo - Perú
}

\section{RESUMEN}

La investigación tuvo por objetivo, establecer un modelo sobre aprendizaje basado en problemas (ABP), para el desarrollo del pensamiento crítico y creativo en estudiantes de cuarto grado de educación primaria de la institución educativa $\mathrm{N}^{\circ} 10828$. La investigación fue de tipo básica, enfoque cuantitativo, nivel propositivo, con el diseño descriptivo propositivo, la población lo conformaron 218 estudiantes y 6 docentes, mientras que la muestra fue determinada con un muestreo probabilístico donde haciendo uso de la fórmula finita se obtuvo una cantidad de 139 estudiantes. Para la recopilación de información se usó un cuestionario de pensamiento crítico y el Test de Torrence para el pensamiento creativo y se administró un cuestionario para los docentes respecto al pensamiento crítico, creativo y a la estrategia $\mathrm{ABP}$, los que fueron validados por juicio de expertos obteniéndose un coeficiente de 0.928 de validez de Aiken. Por resultados se obtuvo que los estudiantes presentaron un nivel medio de pensamiento crítico y un nivel bajo de creatividad. Frente a ello, se diseñó un modelo de aprendizaje basado en problemas (ABP) para fortalecer el pensamiento crítico y creativo. La propuesta fue validada por juicio de expertos, donde los profesionales validadores brindaron su aprobación, con un coeficiente de 0.947 .

Palabras Clave: estrategia; modelo ABP; pensamiento creativo; pensamiento crítico. 


\title{
Problem based learning for critical thinking and creative in elementary school students
}

\begin{abstract}
The objective of the research was to establish a model on problem-based learning (PBL), for the development of critical and creative thinking in students of the fourth grade of primary education of the educational institution $\mathrm{N}^{\circ} 10828$. The research was of a basic type, quantitative approach, propositional level, with a descriptive-propositional design, the population was made up of 218 students and 6 teachers, while the sample was determined with a probability sampling where, using the finite formula, a number of 139 students. For the collection of information, a critical thinking questionnaire and the Torrence Test for creative thinking were used, and a questionnaire was administered for teachers regarding critical and creative thinking and the PBL strategy, which were validated by expert judgment, obtaining a Aiken validity coefficient of 0.928 . By results it was obtained that the students presented a medium level of critical thinking and a low level of creativity. Against this, a problem-based learning (PBL) model was designed to strengthen critical and creative thinking. The proposal was validated by expert judgment, where the validating professionals gave their approval, with a coefficient of 0.947 .
\end{abstract}

Key words: strategy, PBL model, creative thinking, critical thinking

Artículo recibido: 02 enero 2022 Aceptado para publicación: 28 enero 2022 Correspondencia: chimoyle@ucvvirtual.edu.pe Conflictos de Interés: Ninguna que declarar 


\section{INTRODUCCIÓN}

La educación en el mundo entero constituye una preocupación de los gobiernos de los diferentes países buscando la calidad y generalidad de tal forma que todos los habitantes de la tierra sean bien formados como es así que el siglo XXI se constituye en un desafía para la educación y específicamente para los docentes en desarrollar capacidades críticas y creativas en los estudiantes de todos los niveles educativos de tal manera que se cuente con niños y jóvenes capaces de presentar ideas, proyectos hacer enjuiciamientos pasando de lo pasivo a lo activo realizando argumentaciones en el entorno donde se encuentra y así constituirse en un ente relevante para la sociedad.

La ministra de educación de España, Celaá (2019), sostuvo que se debe elaborar un nuevo plan de estudios centrado en las competencias, con la finalidad de desarrollar habilidades, como la creatividad, la comunicación, la cooperación y la criticidad que requiere la era digital.

En Costa Rica, Vargas (2019) enseñar y aprender habilidades de pensamiento de alto nivel es un desafío que debe lanzarse en las escuelas para lograr un aprendizaje activo, promover la comprensión y evitar la aplicación simple y repetitiva de los conocimientos. Los niños dentro de sus primeras experiencias vivenciadas rompen esquemas constantemente, por lo general ellos no tienen una rutina de vida y por naturaleza son inquietos, curiosos, originales y sobre todo creativos, pero dentro de las instituciones se está llevando una educación equivocada limitándolos a seguir desarrollando su creatividad, dado que los docentes tienen una fuerte obsesión con que los niños den respuestas claras y concretas frente a una pregunta frente a diferentes procesos evaluativos que únicamente se centran en el pensamiento lógico racional, dejando de la mejora en el desarrollo de otras actividades (2019).

Jeong (2019), señala que en la educación básica los docentes se rigen a modelos de enseñanza estandarizados que no siempre potencializan el pensamiento crítico y creativo de los niños, generando dificultades en el desarrollo de habilidades creativas y capacidad crítica de los estudiantes, por ello, en muchas instituciones educativas se presenta la necesidad de que los docentes identifiquen sus habilidades de manera personalizada y que se apliquen herramientas acorde a la realidad y necesidad del alumno.

En Perú, la ex ministra de Educación, Pablo (2019) expresó "En este país, la cobertura ha mejorado, pero la calidad no." Indicando que el $90 \%$ de los maestros no desarrollan 
habilidades de razonamiento crítico en sus alumnos y el $91 \%$ no aplica estrategias de retroalimentación para que los alumnos puedan descubrir sus propias brechas y habilidades.

Morante (2019) indicó que las canciones y el recreo son herramientas esenciales para aumentar las habilidades de un niño, a pesar de los progresos tecnológicos, la educación en Perú sigue siendo una tradición. Por tanto, los profesores deben manejar estrategias que refuercen el pensamiento creativo.

A través de la observación directa y a la encuesta aplicada a los docentes, se comprobó que los estudiantes del cuarto grado de educación primaria de la institución educativa $\mathrm{N}^{\circ} 10828$, tienen un bajo nivel en el pensamiento crítico y creativo, porque demuestran poca participación en clase, cierto miedo para responder preguntas, escasa curiosidad sobre un tema a tratar, no hacen cuestionamientos, generalmente no toman decisiones para mostrar creatividades frente a un problema u otra situación, así como muestran exceso de dependencia.

Las causas de este problema son, los docentes no fomentan con periodicidad la investigación y la creatividad, los estudiantes tienen escasas oportunidades de participación en el aprendizaje basado en problemas y su relación con los aspectos críticos y creativos; limitado trabajo en equipo, no se desarrolla el debate, escasa utilización de metodología activa, restringida promoción de las preguntas analíticas alejados del aprendizaje autónomo. Los estudiantes no tienen la oportunidad de trabajar en el ABP Por consiguiente, si el problema precisado no se resuelve, habrá limitaciones para apoyar sobre lo que establece la ODS para el 2030, certificar una enseñanza de eficacia y fomentar oportunidades de estudio a lo largo de toda la vida, y también frente a lo que la OCDE (2018) señala que para el siglo XXI, en la educación se debe suscitar las destrezas entre ellas la creatividad y pensamiento crítico. Entonces se tienen que desarrollar estas habilidades, fundamentalmente durante los primeros años de vida, porque mientras más temprano se fomenten, más eficaces y de calidad serán los aprendizajes.

El propósito de la investigación es proponer un modelo del aprendizaje basado en problemas el cual permita desarrollar el pensamiento crítico y creativo de los estudiantes del cuarto grado de educación primaria de la institución educativa mencionada.

Ante la situación planteada, se formula el siguiente problema; ¿De qué manera el modelo ABP desarrolla el pensamiento crítico y creativo en estudiantes de cuarto grado de 
educación primaria de la institución educativa №10828 ¿Ex Cosome de Chiclayo, 2021? El presente estudio se justifica porque actualmente es fundamental que la criticidad y creatividad se vean incrementadas, estimuladas por el nuevo estilo de vida y contexto mundial, para superar en los educandos de cuarto grado de la institución educativa №10828 de Chiclayo el bajo nivel de desarrollo del pensamiento crítico y creativo, es preciso, fomentar en los educandos las habilidades creativas y de criticidad. La realización de esta investigación contribuirá para que los estudiantes sean personas transformadoras y competentes, promoviendo el progreso social.

Asimismo, tiene como aporte teórico incrementar el conocimiento de las variables por medio de su análisis y frente a ello diseñar un modelo de ABP para el desarrollo del pensamiento crítico y creativo en estudiantes de educación primaria. Además, resulta relevante porque se beneficiará directamente a los estudiantes, luego a los padres de familia y consecuentemente a la comunidad donde está inmersa la institución educativa donde se realizó la investigación.

La investigación tuvo como objetivo general, establecer un modelo sobre aprendizaje basado en problemas para el desarrollo del pensamiento crítico y creativo en estudiantes de cuarto grado de educación primaria de la institución educativa $\mathrm{N}^{\circ} 10828$ Ex Cosome,2021. De igual manera se establecieron tres objetivos específicos, diagnosticar mediante un cuestionario y test de Torrance el nivel del desarrollo del pensamiento crítico y creativo respectivamente en los estudiantes de cuarto grado de la institución educativa $\mathrm{N}^{\circ} 10828$, diseñar un modelo sobre aprendizaje basado en problemas para el desarrollo del pensamiento crítico y creativo en los estudiantes que participaron en la investigación y validar el modelo sobre aprendizaje basado en problemas mediante juicio de expertos. La hipótesis queda formulada de la forma siguiente: El modelo ABP es una estrategia efectiva para el desarrollo del pensamiento crítico y creativo en estudiantes de cuarto grado de educación primaria de la institución educativa $\mathrm{N}^{\circ} 10828$ Ex Cosome, 2021.

\section{ESTRATEGIAS METODOLÓGÍCAS}

\subsection{Tipo y diseño de investigación}

\subsubsection{Tipo de investigación: Descriptiva proyectiva.}

En presente trabajo es de tipo básica, que busca relatar el desarrollo del pensamiento crítico y creativo en los niños de cuarto grado de la Institución Educativa $\mathrm{N}^{\circ} 10828$ Ex Cosome. Asimismo, la vinculación con la estrategia aprendizaje basado en problemas 
para una enseñanza óptima, el objetivo de la investigación es coleccionar los datos e indagaciones sobre el fenómeno que es sometido a un estudio (Ñaupas et al., 2018) La presente investigación en cuestión también es denominada de tipo propositiva, debido a que, al momento de realizar la identificación de los pensamientos críticos y creativos, se realiza el planeamiento de una propuesta sobre el aprendizaje en función en conflictos como alternativa para repotenciar tales capacidades. Por medio de esta propuesta se ofrece argumentos acerca de conceptualización y acciones con la finalidad de recomendar a los educadores su ejecución posterior, tal como lo indica Estela (2020) donde manifiesta que es la consecuencia de la observación y exposición de teorías en base a las variables de estudio para que se pueda desarrollar el diseño de la propuesta, con el propósito de resolver el fenómeno en estudio.

\subsubsection{Diseño de investigación}

El diseño utilizado en la presente investigación es no experimental, de corte transversal, pues en el estudio se buscó recoger información de un fenómeno, donde se hace un diagnóstico y evaluación, después se analizará, finalizando con una propuesta de solución (Tantaleán, 2015)

M------O $\rightarrow \mathrm{D} \rightarrow \mathrm{P}$

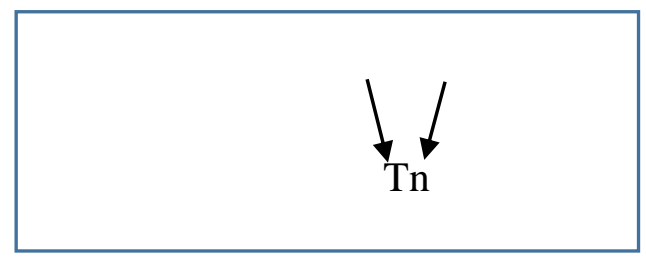

\section{Donde:}

M: Representa la muestra

O: Información que se recogerá de las personas investigadas.

D: Diagnóstico y evaluación

tn: Análisis de las teorías para comprender el fenómeno para elaborar una propuesta

P: Es la propuesta

\subsection{Variables y operacionalización}

2.2.1. Variables dependientes: Pensamiento crítico y creativo

2.2.2. Variable independiente: Aprendizaje basado en problemas 


\subsection{Población, muestra, muestreo}

\subsubsection{Población}

López y Fachelli (2017), "la población son los elementos que constituyen el espacio de utilidad analítico y sobre el que se quiere desprender las conclusiones del análisis” (p.7). La población de estudio está conformada por 218 estudiantes de cuarto grado de Educación Primaria, de la Institución Educativa N¹0828 Ex Cosome - Chiclayo, Conforme se aprecia en la Tabla 1

\section{Tabla 01}

Distribución del número de estudiantes del cuarto grado de la Institución Educativa $N^{\circ} 10828$ Ex Cosome - Chiclayo

\begin{tabular}{cccc}
\hline sección & Hombres & Mujeres & Total \\
\hline A & 15 & 22 & 37 \\
B & 19 & 21 & 40 \\
C & 19 & 19 & 38 \\
D & 18 & 15 & 33 \\
E & 17 & 19 & 36 \\
F & 16 & 18 & 34 \\
\hline Total & 104 & 114 & 218 \\
\hline Docentes & & 6 & 6 \\
\hline
\end{tabular}

Nota: Nómina de matrícula 2021 y CAP de docentes.

\subsubsection{Muestra}

Para establecer la muestra, se utilizó un muestreo aleatorio simple, en la cual se establece que todos los participantes de la población tienen la probabilidad de ser seleccionados. Por lo tanto, para su cálculo se hizo uso de la fórmula de la muestra finita, considerando un $95 \%$ de confiabilidad y un margen de error del 5\%, llegando a obtener una cantidad de 139 niños, los que representarán la muestra de estudio.

\subsection{Técnicas e instrumentos de recolección de datos.}

Se considera a las técnicas de recolección de datos como aquella acción que se realiza para que se logre obtener información oportuna y relevante, mientras que los instrumentos de recolección de datos hacen representación al medio que se usará para alcanzar obtención de información confiable, destacando así que estos tienen que enfocarse en 
lograr medir una situación, por lo tanto tanto las técnicas como los instrumentos tienen que disponer un mismo objetivo (Hernández y Duana, 2020)

Técnica de la observación

La técnica de la observación fue aplicada a través del test "Pensamiento creativo de Torrance - figurativo", evalúa las dimensiones de originalidad, elaboración, flexibilidad y fluidez, asimismo está conformado por un baremo que mide el nivel de creatividad alta de 186 - 278 puntos, media de 93-185 puntos y baja de 00-92, Esta técnica es un método confiable, válido y permite el registro sistemático de la variable o variables a estudiar Hernández (2018)

Técnica de la encuesta

La técnica de la encuesta se utilizó aplicando un cuestionario para medir el pensamiento crítico de los estudiantes, evalúa las dimensiones de análisis, interpretación, juicio e inferencia usando la escala de Likert, también se aplicó un cuestionario a los docentes el cual permitió recolectar información sobre el desarrollo del pensamiento crítico y creativo y si aplican la estrategia del aprendizaje basado en problemas en los estudiantes, Bernal (2016) la encuesta se usa para recopilar información a través de preguntas objetivas y coherentes para garantizar que la información recopilada pueda analizarse.

\section{RESULTADOS}

Luego de haber aplicado los instrumentos de recolección de datos, se organizó la información obtenida mediante tablas y figuras, con el propósito de responder a los objetivos de investigación y detallar la realidad del estudio.

Resultados de la Dimensión Análisis e interpretación

\section{Tabla 2}

Nivel de las dimensiones del pensamiento crítico.

\begin{tabular}{lcccccc}
\hline & \multicolumn{3}{c}{ Dimensiones } \\
\cline { 2 - 7 } & Análisis e interpretación & \multicolumn{2}{c}{ Juicio } & \multicolumn{2}{c}{ Inferencia } \\
\hline Nivel & $\mathrm{f}$ & $\%$ & $\mathrm{f}$ & $\%$ & $\mathrm{f}$ & $\%$ \\
Bajo & 21 & $15 \%$ & 26 & $19 \%$ & 18 & $13 \%$ \\
Medio & 69 & $50 \%$ & 72 & $52 \%$ & 64 & $46 \%$ \\
Alto & 49 & $35 \%$ & 41 & $29 \%$ & 57 & $41 \%$ \\
Total & $\mathbf{1 3 9}$ & $\mathbf{1 0 0 \%}$ & $\mathbf{1 3 9}$ & $\mathbf{1 0 0 \%}$ & $\mathbf{1 3 9}$ & $\mathbf{1 0 0 \%}$ \\
\hline
\end{tabular}

Nota: Elaboración propia. 


\section{Figura 1}

Nivel de las dimensiones del pensamiento crítico.

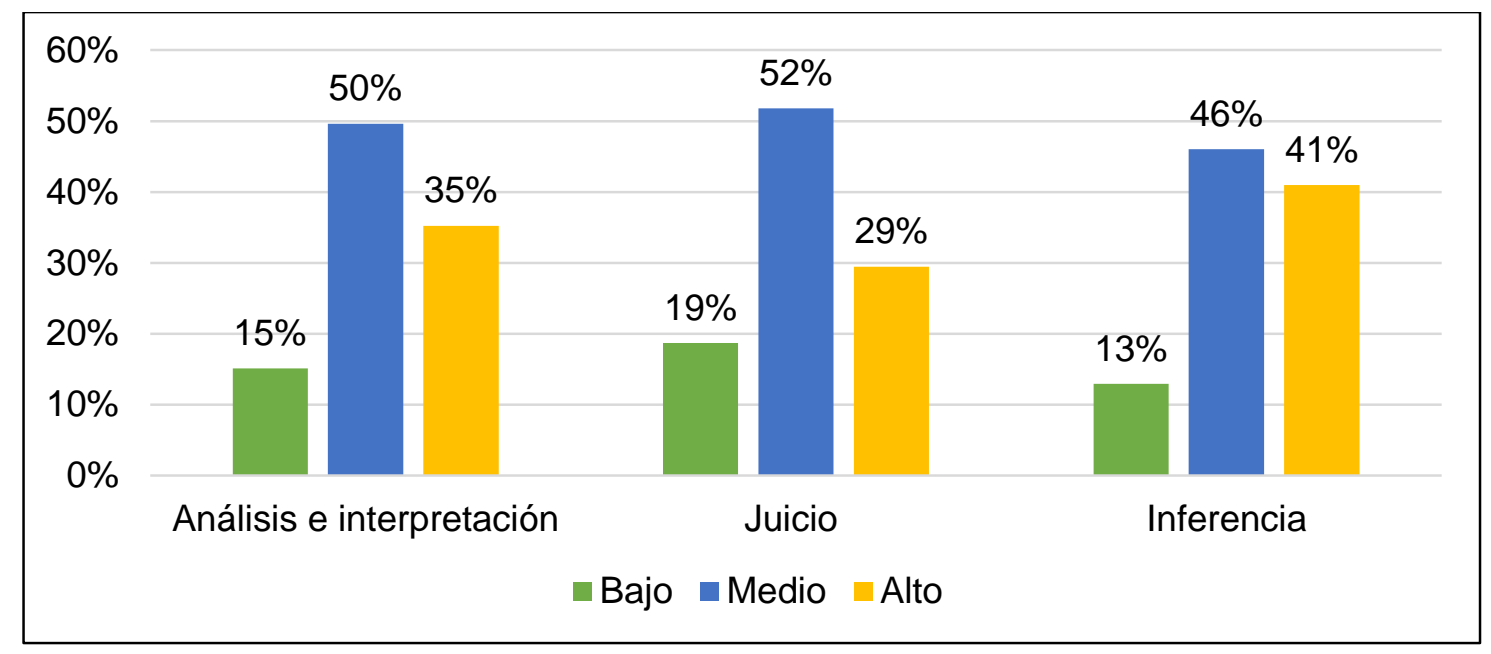

Fuente: elaboración propia.

Interpretación. Efectuando un análisis a los resultados se pudo determinar que respecto a las dimensiones del pensamiento crítico, el 50\% de los estudiantes presenta un nivel medio de análisis e interpretación con tendencia a alto, de igual manera en la dimensión juicio prevalece el nivel medio, por último el $46 \%$ de los estudiantes destaca un nivel medio de inferencia, es de esta manera como en base a los resultados, se puede evidenciar que los alumnos del cuarto grado de educación primaria de la Institución Educativa $\mathrm{N}^{\circ} 10828$ Ex Cosome, presentan la necesidad de potencializar sus capacidades de análisis crítico, inferencia y juicio, para que de esta manera se fortalezca su pensamiento crítico, mejorando así su capacidad de análisis e interpretación.

\subsection{Resultados sobre el pensamiento crítico}

\section{Tabla 3}

Nivel del pensamiento crítico.

\begin{tabular}{ccc}
\hline Nivel & Frecuencia & Porcentaje \\
\hline Bajo & 22 & $16 \%$ \\
Medio & 68 & $49 \%$ \\
Alto & 49 & $35 \%$ \\
Total & $\mathbf{1 3 9}$ & $\mathbf{1 0 0 \%}$ \\
\hline
\end{tabular}

Nota: Elaboración propia. 


\section{Figura 2}

Nivel del pensamiento crítico.

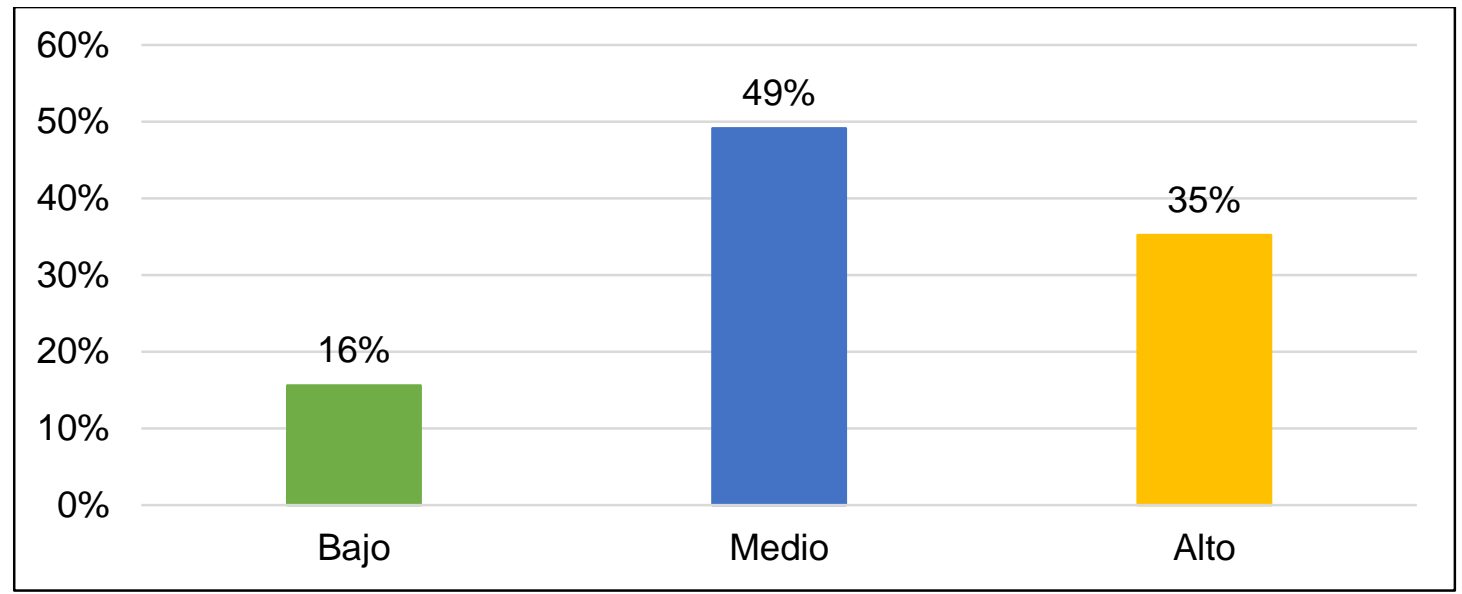

Fuente: elaboración propia.

Interpretación. Concerniente a los resultados presentados, se logra enfatizar que el $49 \%$ los estudiantes del cuarto grado de educación primaria de la Institución Educativa $\mathrm{N}^{\circ} 10828$ Ex Cosome presentan un nivel medio de pensamiento crítico, por lo cual se indica, que los docentes necesita potencializar sus estrategias educativas alineadas a la necesidad de fortalecer el pensamiento crítico de los estudiantes, situación que favorecerá a mejorar el rendimiento académico del alumno en todos los niveles y cursos académicos.

\subsection{Resultados sobre las dimensiones del pensamiento creativo}

\section{Tabla 4}

Nivel de las dimensiones del pensamiento creativo.

\begin{tabular}{|c|c|c|c|c|c|c|c|c|}
\hline \multirow[b]{3}{*}{ Nivel } & \multicolumn{8}{|c|}{ Dimensiones } \\
\hline & \multicolumn{2}{|c|}{ Flexibilidad } & \multicolumn{2}{|c|}{ Fluidez } & \multicolumn{2}{|c|}{ Originalidad } & \multicolumn{2}{|c|}{ Elaboración } \\
\hline & $\mathrm{f}$ & $\%$ & $f$ & $\%$ & $\mathrm{f}$ & $\%$ & $\mathrm{f}$ & $\%$ \\
\hline Bajo & 72 & $52 \%$ & 78 & $56 \%$ & 65 & $47 \%$ & 73 & $53 \%$ \\
\hline Medio & 39 & $28 \%$ & 42 & $30 \%$ & 56 & $40 \%$ & 44 & $32 \%$ \\
\hline Alto & 28 & $20 \%$ & 19 & $14 \%$ & 18 & $13 \%$ & 22 & $15 \%$ \\
\hline Total & 139 & $100 \%$ & 139 & $100 \%$ & 139 & $100 \%$ & 139 & $100 \%$ \\
\hline
\end{tabular}

Nota: Elaboración propia. 


\section{Figura 3}

Nivel de las dimensiones del pensamiento creativo.

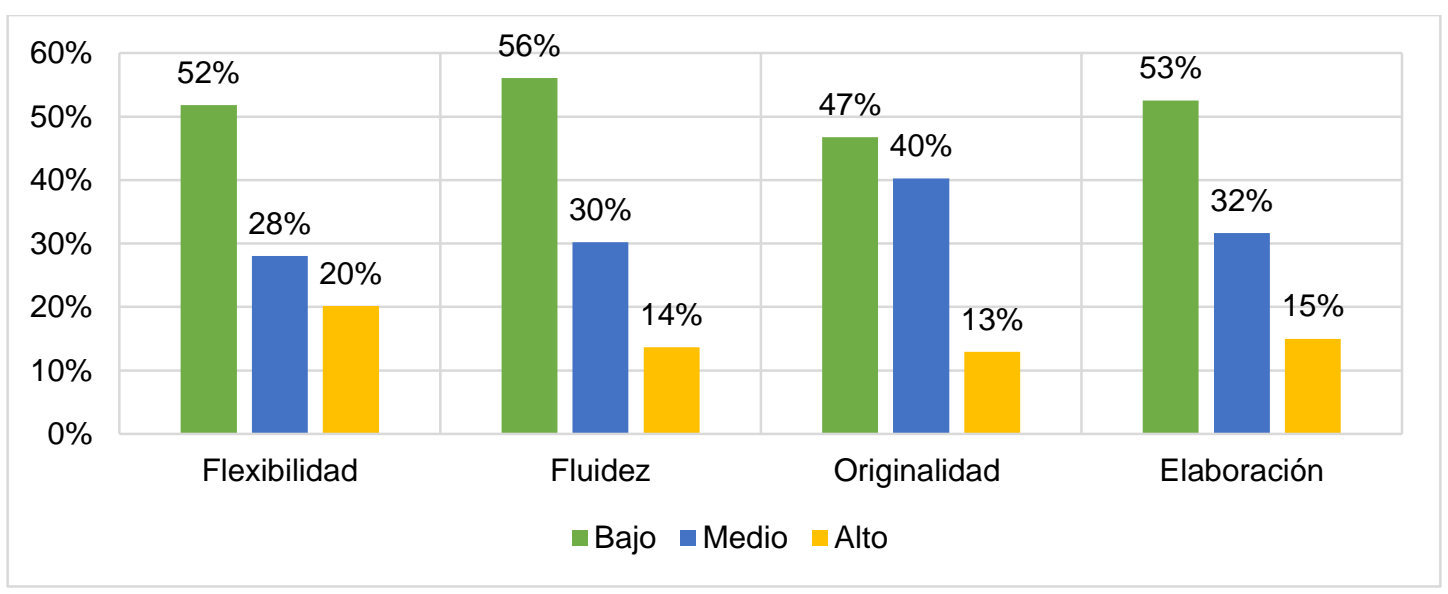

Fuente:

Interpretación. En la presentación de los resultados, respecto al análisis de las dimensiones del pensamiento crítico, el 52\% de los estudiantes de la Institución Educativa $\mathrm{N}^{\circ} 10828$ Ex Cosome presenta un nivel bajo de la dimensión flexibilidad, destacando que los estudiantes presentan resistencia al cambio, no se acomodan con facilidad a condiciones diferentes, de igual manera el $56 \%$ de los alumnos presentaron un nivel bajo de fluidez, lo que determina dificultad de los estudiantes para producir ideas, asimismo, $47 \%$ presentó un nivel bajo de originalidad, situación que demuestra que los estudiantes con mayor facilidad se acoplan a lo que ya conoce y se resisten a desarrollar situaciones propias, que los identifique como niños únicos. Por último, el 53\% de los estudiantes presentaron un nivel bajo de elaboración, lo que denota, que los alumnos presentan dificultad para organizar, sistematizada o seleccionar todo en un orden de ideas creativas, es de esta forma como se puede evidenciar unos alumnos poseen bajos niveles de creatividad.

\subsection{Resultados sobre el pensamiento creativo}

\section{Tabla 5}

Nivel del pensamiento creativo.

\begin{tabular}{ccc}
\hline Nivel & Frecuencia & Porcentaje \\
\hline Bajo & 72 & $51 \%$ \\
Medio & 45 & $33 \%$ \\
Alto & 22 & $16 \%$ \\
Total & $\mathbf{1 3 9}$ & $\mathbf{1 0 0 \%}$ \\
\hline
\end{tabular}

Nota: Elaboración propia. 


\section{Figura 4}

Nivel del pensamiento creativo.

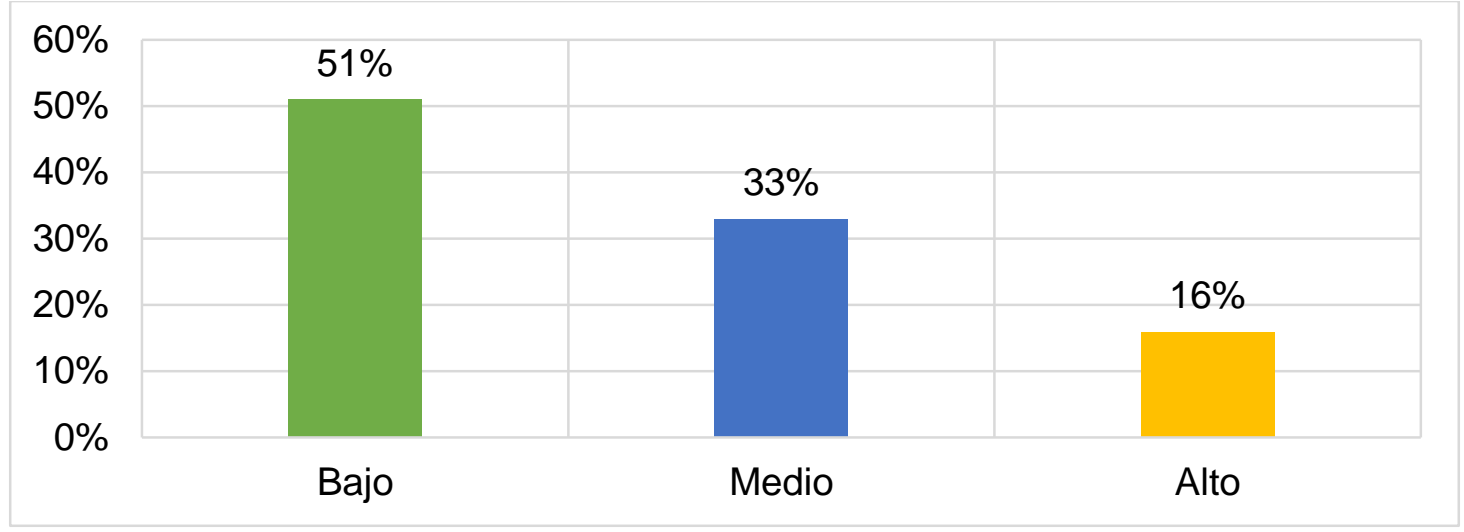

Fuente: elaboración propia.

Interpretación.Tomando referencia a los resultados obtenidos se destaca que más del $50 \%$ de los estudiantes de la Institución Educativa $\mathrm{N}^{\circ} 10828$ Ex Cosome presentan un nivel bajo de creatividad, situación que demuestra la necesidad de que los docentes que implementen estrategias pedagógicas para crear condiciones idóneas innecesarias en estimulación y desarrollo de todas las facultades activas de los alumnos, inculcando un aprendizaje abierto y flexible, se requiere que se rompan los paradigmas y adecuar su proceso de enseñanza hacia la necesidad de cada estudiante pues ello favorecerá a mejorar potencializar su capacidad creativa.

\subsection{Consolidado de resultados a nivel del pensamiento crítico y creativo}

\section{Tabla 6}

Consolidado de resultados a nivel del pensamiento crítico y creativo.

\begin{tabular}{ccc}
\hline Nivel & Frecuencia & Porcentaje \\
\hline Bajo & 47 & $34 \%$ \\
Medio & 57 & $41 \%$ \\
Alto & 35 & $25 \%$ \\
Total & $\mathbf{1 3 9}$ & $\mathbf{1 0 0 \%}$ \\
\hline
\end{tabular}

Nota: Elaboración propia. 


\section{Figura 5}

Consolidado de resultados a nivel del pensamiento crítico y creativo.

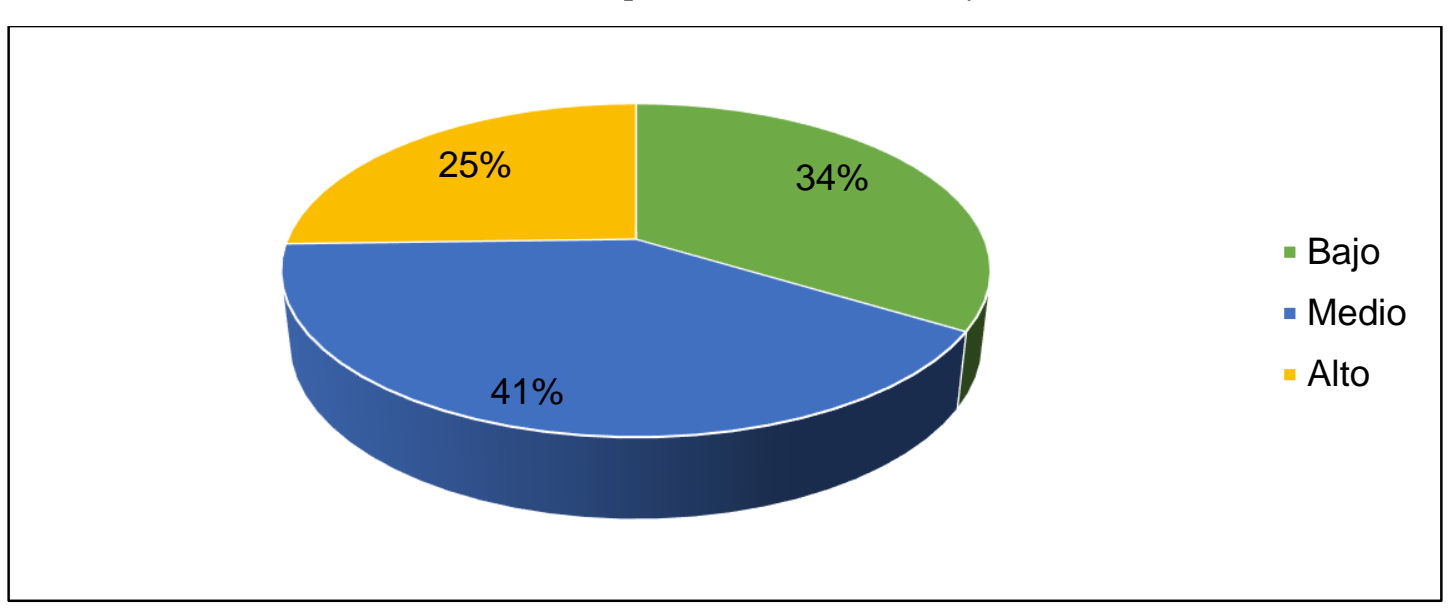

Fuente:

Interpretación.Resaltando los resultados encontrados se llega a establecer que el $41 \%$ de los estudiantes destacan un nivel medio de pensamiento crítico y creativo, ese esta manera como se infiere la necesidad que presentan los alumnos para abrirse al cambio y desarrollar sus propias habilidades en construcción de diagramas, creación de cosas y sobre todo su análisis crítico e inferencial. De igual manera, se presenta la necesidad de que los docentes aplicar nuevas estrategias críticas y creativas que ayuden al alumno a desenvolverse de manera más original y propia en diferentes ambientes, favoreciendo así su capacidad de adaptación y relación con la sociedad.

\section{DISCUSIÓN}

La investigación estuvo orientada a establecer un modelo sobre aprendizaje basado en problemas para el desarrollo del pensamiento crítico y creativo en estudiantes de cuarto grado de educación primaria de la institución educativa $\mathrm{N}^{\circ} 10828$ Ex Cosome, por tal razón es de suma importancia la aplicación del modelo $\mathrm{ABP}$, porque permitirá el fortalecimiento de las habilidades de criticidad y creatividad.

Para tener conocimiento de los niveles del pensamiento crítico y creativo que presentan los estudiantes, se elaboró dos test, los cuales fueron validados por cinco juicios de expertos y posteriormente se midió la confiabilidad a través de una prueba piloto, finalmente se aplicó a la muestra de estudio de la investigación, obteniendo los siguientes resultados.

Efectuando un análisis a los objetivos de investigación se destaca que respecto al estudio de la variable del pensamiento crítico se llegó a identificar que el 49\% los estudiantes del 
cuarto grado de educación primaria de la Institución Educativa №10828 Ex Cosome presentan un nivel medio de pensamiento crítico, por lo cual se indica, que los docentes necesita potencializar sus estrategias educativas alineadas a la necesidad de fortalecer el pensamiento crítico de los estudiantes, situación que favorecerá a mejorar el rendimiento académico del alumno en todos los niveles y cursos académicos.

De igual forma, respecto al análisis que se ha efectuado a las dimensiones del pensamiento crítico se puede identificar el 50\% de los estudiantes presenta un nivel medio de análisis e interpretación con tendencia a alto, de igual manera en la dimensión juicio prevalece el nivel medio, por último el $46 \%$ de los estudiantes destaca un nivel medio de inferencia, es de esta manera como en base a los resultados, se puede evidenciar que los alumnos del cuarto grado de educación primaria de la Institución Educativa $\mathrm{N}^{\circ} 10828$ Ex Cosome, presentan la necesidad de potencializar sus capacidades de análisis crítico, inferencia y juicio, para que de esta manera se fortalezca su pensamiento crítico, mejorando así su capacidad de análisis e interpretación.

Estos resultados se llegan a contrastar con los estudios de Manayay (2018),quién en su investigación llegó a establecer que el 55\% de los estudiantes presentan un nivel regular de pensamiento crítico, el $40 \%$ destacó un nivel deficiente y solo un 5\% referenció un nivel bueno, el autor llega a concluir que son porcentajes alarmantes pues el que los estudiantes dispongan de bajos niveles de pensamiento crítico conllevará a que no tengan un adecuado rendimiento escolar, Por otro lado enfatizó que luego de haber aplicado un modelo de ABP esto influenció de manera significativa en la mejoría de su nivel de pensamiento crítico favoreciéndolo hasta el $95 \%$ de los estudiantes, destacando que la implementación de estrategias son de gran importancia para el desarrollo académico e intelectual de los estudiantes.

De manera similar se contrasta con la investigación realizada por Arévalo (2020), quien presentó el $55 \%$ de los estudiantes destacaron un nivel medio de pensamiento crítico mientras que el $26 \%$ presentó un nivel bajo y un $19 \%$ destacó un nivel alto, porcentajes que también captaron la atención del investigador pues la principal problemática de su estudio fue el bajo nivel de comprensión lectora, en la cual el pensamiento crítico es requisito fundamental para que este nivel de comprensión se fortalezca, es así como del estudio se logra rescatar que los docentes al alinear sus estrategias y metodologías de enseñanza en el pensamiento crítico de los alumnos esto no solamente favorecerá a su 
análisis inferencial, literal y crítico sino que también favorecerá al desenvolvimiento de todas las áreas académicas.

Por el contrario se difiere con el estudio presentado por Clemens (2016), pues en su investigación obtuvo altos niveles de pensamiento crítico en los estudiantes de sexto grado de primaria, sin embargo rescató un 30\% que aún se encuentra en un nivel medio de pensamiento crítico, este autor llega a concluir qué respecto a la implementación de estrategias didácticas de aprendizaje colaborativo llega a fomentar el pensamiento crítico de los estudiantes pues destaca que esta capacidad es la fuente principal que permite que los estudiantes infieran de manera autónoma al análisis crítico ya sea de textos o situaciones problemáticas favoreciendo directamente a la toma de decisiones.

Además se llega a contrastar, con el estudio establecido por Paredes (2016), quien indica en su investigación que los estudiantes desarrollan un pensamiento crítico reflexivo medio, ello destaca que por medio de la implementación de modelos didácticos holísticos divergentes ayudar a que se mejore progresivamente la capacidad crítica de los estudiantes y esto les favorecerá al desarrollo de la condición autocrítica frente a las diferentes situaciones que se les presentan en su vida cotidiana, es así que ellos dispondrán de una actitud de confianza en sus conocimientos para innovar.

En lo que concierne al análisis del pensamiento creativo se logró enfatizar que el $51 \%$ de los estudiantes del cuarto grado de educación primaria de la Institución Educativa $\mathrm{N}^{\circ} 10828$ Ex Cosome presentaron un nivel bajo de creatividad, situación que demuestra la necesidad de que los docentes que implementen estrategias pedagógicas para de esta forma generar la creación de ideas y condiciones idóneas innecesarias en estimulación y desarrollo de todas las facultades activas de los alumnos, inculcando un aprendizaje abierto y flexible, se requiere que se rompan los paradigmas y adecuar su proceso de enseñanza hacia la necesidad de cada estudiante pues ello favorecerá a mejorar potencializar su capacidad creativa.

En un análisis a sus dimensiones también se llegó a establecer, el 52\% de los estudiantes poseen un nivel bajo de flexibilidad creativa, el $56 \%$ presenta un nivel bajo de fluidez, el $47 \%$ nivel bajo de originalidad creativa y el 53\% del nivel bajo de elaboración, es así que en base a los resultados manifestado se presenta la necesidad de que los docentes implementen estrategias que se alineen a fortalecer estas dimensiones pues por medio de 
un adecuado proceso de enseñanza aprendizaje conllevará a que los estudiantes fortalezcan cada una de estas capacidades.

Así como estos resultados se llegan a contrastar con el estudio de Arévalo (2020), quién es su investigación llegó a concluir que el 29\% de los estudiantes presentaron un nivel medio del pensamiento creativo, encontrando diferencia con los resultados presentados, sin embargo el autor también llegó a establecer que el pensamiento creativo presenta una relación significativa con la comprensión lectora, es decir que si se potencializa esta capacidad los estudiantes llegarán a poseer mejores niveles de comprensión.

De igual forma se encuentra similitud con los resultados de Palmer (2019), quien indica que el pensamiento creativo no solamente se alinea a la creatividad de los estudiantes sino a la forma en como los docentes imparten sus enseñanzas, es así quienes estudio estableció que el 50\% de los docentes llegaron a establecer que si ellos Fortalecen su potencial creativo, esto influenciará de manera significativa en el aprendizaje de los estudiantes y con ello se potencializará su creatividad, cabe destacar, que las estrategias implementadas siempre tienen que alinearse a la necesidad y realidad de los estudiantes. Por otro lado, se llegó a establecer tengo una agrupación de resultados del pensamiento crítico y creativo, se estableció que el $41 \%$ de los estudiantes destacan un nivel medio de pensamiento crítico y creativo, ese esta manera como se infiere la necesidad que presentan los alumnos para abrirse al cambio y desarrollar sus propias habilidades en construcción de diagramas, creación de cosas y sobre todo su análisis crítico e inferencial. De igual manera, se presenta la necesidad de que los docentes aplicar nuevas estrategias críticas y creativas que ayuden al alumno a desenvolverse de manera más original y propia en diferentes ambientes, favoreciendo así su capacidad de adaptación y relación con la sociedad.

Es de esta forma, cómo se coincide con el estudio de Yapo (2020), quien indica el 21\% de los docentes establecieron que sus estudiantes llegan a desarrollar un nivel de pensamiento crítico alto cuando en el proceso de sus enseñanzas aplican estrategias innovadoras y poco rutinario, por el contrario $57 \%$ de los profesionales señaló que si las estrategias se llegan a implementar de forma regular esto también conllevará a que el nivel de pensamiento crítico de los estudiantes se presente como un nivel medio, indicando que la construcción del pensamiento crítico presenta una relación significativa con la potenciación de las estrategias que apliquen los docentes. 
Por otro lado, que contrasta con el estudio de Salazar y Cabrera (2019), quien destaco que los estudiantes presentan un nivel inferior de capacidad crítica esto debido a que los docentes les cuesta realizar actividades de aprendizaje como escenarios reales en la cual el estudiante muestra con facilidad sus habilidades de pensar en criticidad, Por otro lado destaca que una de las principales características que limitan el desarrollo del pensamiento crítico es la timidez que tienen los estudiantes, es por eso la ardua tarea que tiene la plana docente para capacitarse y poder llegar sus enseñanzas hacia los alumnos para que así potencialicen su capacidad de interpretación y libertad crítica.

de igual manera se llega a aceptar la posición establecida por Castro, Cortazar y Pérez (2018) quienes señalan que el pensamiento crítico es aquella habilidad que las personas desarrollan a medida de su crecimiento profesional y de estudios, y que a través de la cual les permite realizar un proceso de toma de decisiones acertado, debido a la capacidad decisiva que ha ganado a partir del crecimiento en conocimientos y experiencias personales y profesionales.

Asimismo, se acepta la posición de Lara, Avila y Olivares (2017), quienes indican que en todo momento los docentes deben mantener la innovación en su proceso de enseñanza para captar la atención de forma continua del estudiante y éste pueda desarrollar su curiosidad y ganas de indagar e investigar lo que no comprende.

Es así cómo se llega a establecer que la propuesta de un modelo ABP, permitirá el fortalecimiento del pensamiento crítico y creativo de los estudiantes de la institución educativa $\mathrm{N}^{\circ} 10828$-Chiclayo.

\section{CONCLUSIONES}

En el diagnóstico del pensamiento crítico, se pudo establecer el $48 \%$ de los estudiantes del cuarto grado de educación primaria de la Institución Educativa $\mathrm{N}^{\circ} 10828$ Ex Cosome presentaron un nivel medio de pensamiento crítico, asimismo en el análisis a las dimensiones, se obtuvo que el 50\% denoto un nivel medio de análisis e interpretación, el $52 \%$ un nivel medio de juicio y el $46 \%$ también señaló un nivel medio de inferencia, indicando que los docentes necesitan potencializar sus estrategias educativas y que éstas se alineen a fortalecer el pensamiento crítico de los estudiantes.

Analizando el pensamiento creativo, se destacó el $51 \%$ de los estudiantes del cuarto grado de educación primaria de la Institución Educativa $N^{\circ} 10828$ Ex Cosome presentaron un nivel bajo de creatividad, de igual forma en el diagnóstico de las dimensiones se obtuvo 
el $52 \%$ tiene un nivel bajo de flexibilidad en la creatividad, el 56\% y $47 \%$ indicaron un nivel bajo en la fluidez y originalidad creativa, de manera similar al 53\% también denoto un nivel bajo de elaboración, resultados que reflejan la necesidad de que los docentes implementen estrategias pedagógicas que estimulen que busquen el desarrollo de las facultades activas y creativas de los estudiantes, buscando en todo momento que el aprendizaje sea abierto, innovador y flexible.

Se diseñó un modelo de aprendizaje basado en problemas para fortalecer el desarrollo del pensamiento crítico y creativo de los estudiantes del cuarto grado de primaria de la institución educativa número $\mathrm{N}^{\circ} 10828$ Ex Cosome, él mismo que fue desarrollado por medio del planteamiento de 6 sesiones de aprendizaje, alineadas a potencializar el análisis e interpretación, el juicio y la inferencia de los estudiantes, de igual forma, a mejorar las capacidades de originalidad, elaboración, flexibilidad y fluidez de la creatividad.

La propuesta "Programa de aprendizaje basado en problemas para el pensamiento crítico y creativo" fue validada por medio de juicio de expertos, para la cual se tuvo en consideración hacer uso de la técnica DELPHI, la que permitió que los profesionales validadores logré por medio de indicadores brindar el consenso y aprobación el modelo planteado en la investigación. 


\section{PROPUESTA DEL MODELO}

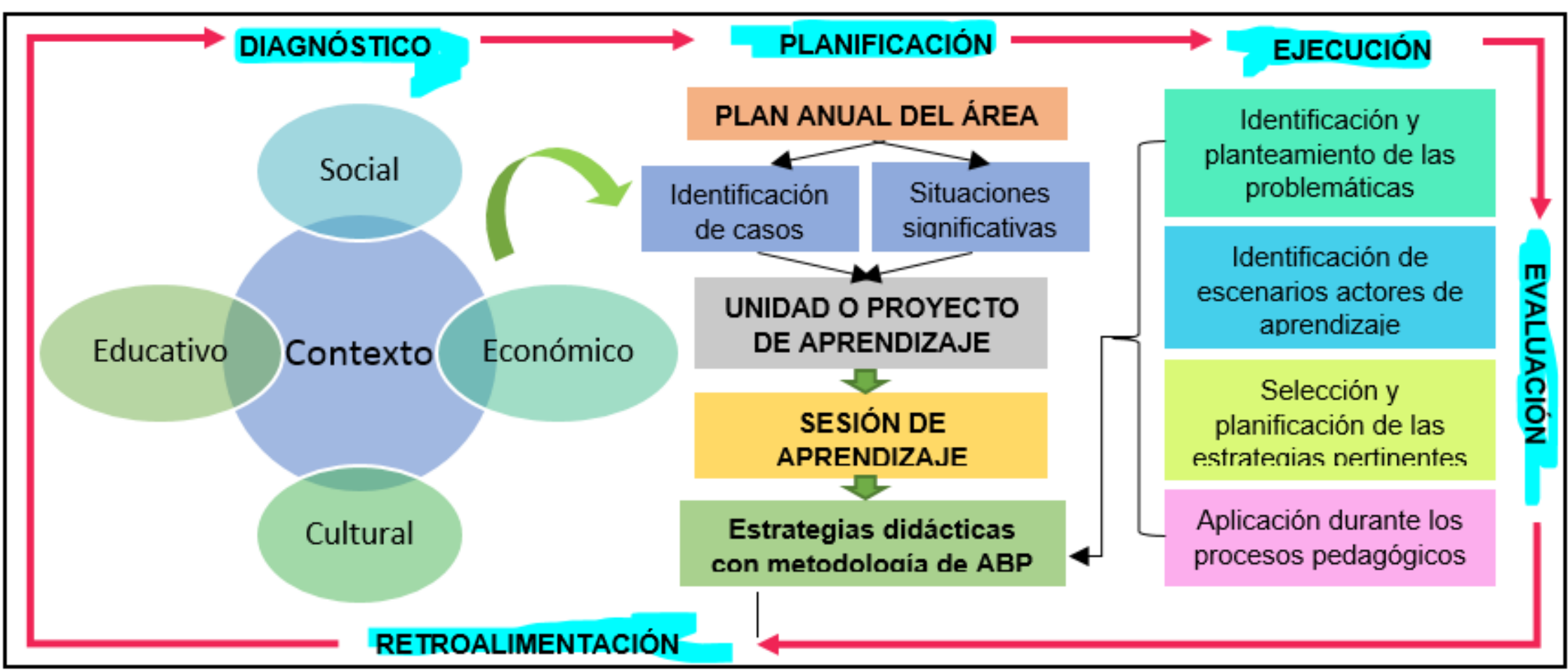




\section{REFERENCIAS BIBLIOGRAFICAS}

Arévalo, T. (2020). Pensamiento creativo y crítico en la comprensión lectora de los estudiantes de primaria de una institución privada, Lince 2020. Lima: Publicaciones de la Universidad César Vallejo. Obtenido de https://repositorio.ucv.edu.pe/handle/20.500.12692/51218

Bernal, C. (2016). Metodología de la investigación. Person Educación

Celaá, I. (2019, 26 de noviembre). El impulso del pensamiento crítico del alumnado y el fomento de una educación para la ciudadanía digital que garantice el uso responsable de internet y de las nuevas tecnologías. http://www.educacionyfp.gob.es/prensa/actualidad/2019/11/20191126consejoeuropa.html

Clemens, A. M. (2016). Desarrollo del pensamiento crítico mediante el aprendizaje colaborativo en alumnos de primaria. [tesis de doctorado, Universidad Complutense de Madrid]. Repositorio Institucional. https://repositorio.tec.mx/bitstream/handle/11285/621385/02ana?sequence=1

Estela, R. (2020). Investigación propositiva. Venezuela: Campus virtual. doi:https://es.calameo.com/read/006239239f8a941bec906

Hernández, S., \& Duana, D. (2020). Técnicas e instrumentos de recolección de datos. Boletín Científico de las Ciencias Económico Administrativas del ICEA, 9(17). Obtenido de https://doi.org/10.29057/icea.v9i17.6019

Hernández-Sampieri, R., \& Mendoza, C. P. (2018). Metodologìa de la Investigaciòn. Las rutas Cuantitativa Cualitativa y Mixta. In Metodología de la investigación. Las rutas cuantitativa, cualitativa y mixta.

Lara, V., Avila, J., \& Olivares, S. (2017). Desarrollo del pensamiento crítico mediante la aplicación del Aprendizaje Basado en Problemas. Scielo; Psicologia Escolar e $\begin{array}{lll}\text { Educacional, } & \text { SP, } & \text { 65-77. }\end{array}$ doi:https://www.scielo.br/j/pee/a/P5JJjM6Rd9zrnh7HxpRQnqH/?lang=es\&form at $=$ pdf

López, N. (2020). Pensamiento crítico latinoamericano en tiempos de colapso. Revista Internacional de Filosofía y Teoría Social, 25(89), 98-107.

Manayay, M. (2018). Programa de estrategia de Aprendizaje Basado enProblemas para desarrollar el pensamiento crítico en las asignaturas teórico-prácticas de los 
estudiantes del sexto ciclo de Enfermería de la Universidad "Señor de Sipán". Chiclayo: Publicaciones de la Unidersidad César Vallejo. Obtenido de https://repositorio.ucv.edu.pe/bitstream/handle/20.500.12692/31777/manayay_m m.pdf? sequence $=1$

Morante, P. (2019, 09 de Julio). La música y el juego permiten un desarrollo más integral. https://www.laindustriadechiclayo.pe/noticia/1565668143-la-musica-y-el-juegopermiten-un-desarrollo-mas-integral-

Ñaupas, H., Valdivia, M., Palacios, J., \& Romero, H. (2018). Metodología de la investigación. Cuantitativa - Cualitativa, Redacción de la tesis (Quinta ed.). Bogotá: Ediciones de la U. Obtenido de https://corladancash.com/wpcontent/uploads/2020/01/Metodologia-de-la-inv-cuanti-y-cuali-HumbertoNaupas-Paitan.pdf

Pablo, F. (2019, 25 de septiembre). Educación de calidad: ¿cómo avanza el Perú en este desafío?. https://elcomercio.pe/especial/perusostenible/prosperidad/educacioncalidad-como-avanza-peru-este-desafio-noticia-1994632 Jeong, J. (2019). Resiliencia y creatividad en escolares de primaria. Revista De Investigación En Psicología, 22(1), 67-78. doi:https://doi.org/10.15381/rinvp.v22i1.16582

Palmer, E. (2019). Potencial creativo docente y desarrollo del pensamiento crítico en estudiantes del nivel primario de la Institución Educativa 11030 Monsefú. Chiclayo: Publicaciones de la Universidad César Vallejo. Obtenido de https://repositorio.ucv.edu.pe/handle/20.500.12692/56519

Paredes, F. L. (2016). Modelo didáctico Holístico Divergente (HD) para desarrollar el pensamiento crítico reflexivo en estudiantes de segundo grado de una Institución Educativa Primaria de Pisco- Ica. [tesis de doctorado, Universidad César Vallejo]. Repositorio Institucional. https://hdl.handle.net/20.500.12692/19215.

Salazar, D, y Cabrera, X. (2019). Estrategia didáctica para fortalecer el pensamiento crítico en estudiantes de tercer grado de primaria en Institución Educativa de Chiclayo. Revista Científica Institucional TZHOECOEN. https://doi.org/10.26495/tzh.v12i1.1240

Tantaleán, M. (2015). The scope of legal research. Revista de Investigación Jurídica, 11(11), 221-236. Obtenido de 
http://mail.upagu.edu.pe/files_ojs/journals/6/articles/133/submission/copyedit/13 3-13-458-1-9-20151124.pdf

UNESCO. (12 de Mayo de 2017). Educación para los Objetivos del desarrollo Sostenible. https://www.oneplanetnetwork.org

Vargas, M. (2019, 27 de mayo). El desarrollo de habilidades de pensamiento superior, un reto para las escuelas y colegios y colegios costarricenses. https://www.historiauned.net/profesor/editar/268-compartiendo-experienciaseducativas-el-desarrollo-de-habilidades-de-pensamiento-superior-un-reto-paralas-escuelas-y-colegios-costarricenses

Yapo, J. (2020). La construcción del pensamiento crítico y el fortalecimiento de estrategias docentes de la institución educativa $N^{\circ} 7221$ La Rinconada. Lima: Publicaciones de la Universidad César Vallejo. Obtenido de https://repositorio.ucv.edu.pe/handle/20.500.12692/46506 